\title{
High-energy cosmic rays measured with KASCADE-Grande
}

\author{
A. Haungs ${ }^{* 1}$, W.D. Apel ${ }^{1}$, J.C. Arteaga-Velázquez ${ }^{2}$, K. Bekk ${ }^{1}$, M. Bertaina ${ }^{3}$, \\ J. Blümer ${ }^{1,4}$, H. Bozdog ${ }^{1}$, I.M. Brancus ${ }^{5}$, E. Cantoni ${ }^{3,6, a}$, A. Chiavassa ${ }^{3}$, \\ F. Cossavella ${ }^{4, b}$, K. Daumiller ${ }^{1}$, V. de Souza ${ }^{7}$, F. Di Pierro ${ }^{3}$, P. Doll ${ }^{1}$, R. Engel ${ }^{1}$, \\ J. Engler ${ }^{1}$, B. Fuchs ${ }^{4}$, D. Fuhrmann ${ }^{8, c}$, H.J. Gils ${ }^{1}$, R. Glasstetter ${ }^{8}$, C. Grupen ${ }^{9}$, \\ D. Heck ${ }^{1}$, J.R. Hörandel ${ }^{10}$, D. Huber ${ }^{4}$, T. Huege ${ }^{1}$, K.-H. Kampert ${ }^{8}$, D. Kang ${ }^{4}$, \\ H.O. Klages ${ }^{1}$, K. Link ${ }^{4}$, P. Łuczak ${ }^{11}$, M. Ludwig ${ }^{4}$, H.J. Mathes ${ }^{1}$, H.J. Mayer ${ }^{1}$, \\ M. Melissas ${ }^{4}$, J. Milke ${ }^{1}$, B. Mitrica ${ }^{5}$, C. Morello ${ }^{6}$, J. Oehlschläger ${ }^{1}$, \\ S. Ostapchenko ${ }^{1, d}$, N. Palmieri ${ }^{4}$, M. Petcu ${ }^{5}$, T. Pierog ${ }^{1}$, H. Rebel ${ }^{1}$, M. Roth ${ }^{1}$, \\ H. Schieler ${ }^{1}$, S. Schoo ${ }^{1}$, F.G. Schröder ${ }^{1}$, O. Sima ${ }^{12}$, G. Toma ${ }^{5}$, G.C. Trinchero ${ }^{6}$, \\ H. Ulrich ${ }^{1}$, A. WeindI ${ }^{1}$, D. Wochele ${ }^{1}$, J. Wochele ${ }^{1}$, J. Zabierowski ${ }^{11}$ - \\ KASCADE-Grande Collaboration \\ ${ }^{1}$ Institut für Kernphysik, KIT - Karlsruher Institut für Technologie, Germany \\ ${ }^{2}$ Universidad Michoacana, Instituto de Física y Matemáticas, Morelia, Mexico \\ ${ }^{3}$ Dipartimento di Fisica, Università degli Studi di Torino, Italy \\ ${ }^{4}$ Institut für Experimentelle Kernphysik, KIT - Karlsruher Institut für Technologie, Germany \\ ${ }^{5}$ National Institute of Physics and Nuclear Engineering, Bucharest, Romania \\ ${ }^{6}$ Osservatorio Astrofisico di Torino, INAF Torino, Italy \\ ${ }^{7}$ Universidade São Paulo, Instituto de Física de São Carlos, Brasil \\ ${ }^{8}$ Fachbereich Physik, Universität Wuppertal, Germany \\ ${ }^{9}$ Department of Physics, Siegen University, Germany \\ ${ }^{10}$ Dept. of Astrophysics, Radboud University Nijmegen, The Netherlands \\ ${ }^{11}$ National Centre for Nuclear Research, Department of Cosmic Ray Physics, Lodz, Poland \\ 12 Department of Physics, University of Bucharest, Bucharest, Romania \\ ${ }^{a}$ now at: Istituto Nazionale di Ricerca Metrologia, INRIM, Torino; ${ }^{b}$ now at: DLR, Oberpfaffenhofen, Germany; \\ ${ }^{c}$ now at: University of Duisburg-Essen, Duisburg, Germany; ${ }^{d}$ now at: University of Trondheim, Norway. \\ E-mail: andreas.haungs@kit.edu
}

The detection of high-energy cosmic rays above a few hundred $\mathrm{TeV}$ is realized by the observation of extensive air-showers. By using the multi-detector setup of KASCADE-Grande, the energy spectrum and the elemental composition of high-energy cosmic rays in the primary energy range of $10^{14}-2 \cdot 10^{18} \mathrm{eV}$ are investigated. In addition, the large, high-quality data set permits distinct tests of the validity of hadronic interaction models used in interpreting the air-shower measurements. The KASCADE-Grande experiment terminated measurements end of 2012. This contribution gives an overview of the most recent results of the data analysis, and will report about the status of KCDC, the 'KASCADE Cosmic-ray Data Center', where via a web-based interface the data of this experiment will be made available for the interested public.

The European Physical Society Conference on High Energy Physics -EPS-HEP2013, 18-24 July 2013, Stockholm, Sweden

\footnotetext{
*Speaker.
} 


\section{KASCADE-Grande}

Extensive air showers (EAS) are generated when high-energy cosmic particles enter the Atmosphere. Forward-boosted secondary particles as well as emitted light during the development of the EAS in various frequency ranges form the detectable products; see figure 1. Main parts of the experiment are the Grande array spread over an area of $700 \times 700 \mathrm{~m}^{2}$, the original KASCADE array covering $200 \times 200 \mathrm{~m}^{2}$ with unshielded and shielded detectors, a large-size hadron calorimeter, and additional muon tracking devices (fig. 1).
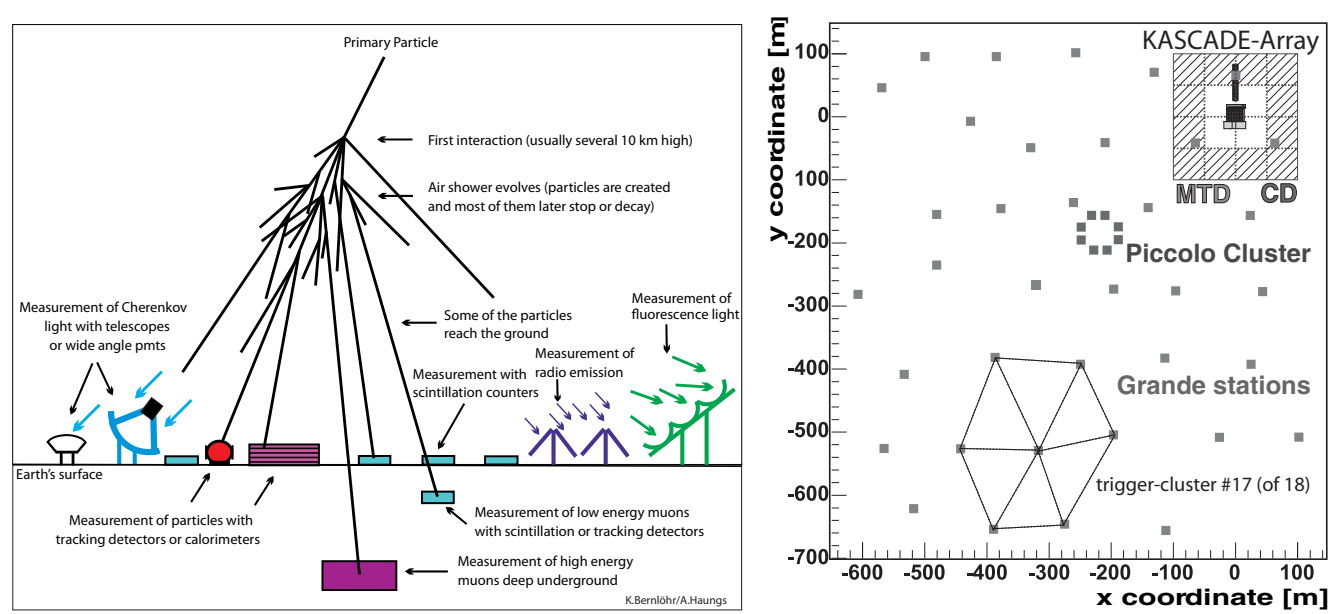

Figure 1: Left: Schematic view of an extensive air-shower with its different detection possibilities. Right: Layout of the KASCADE-Grande experiment: The original KASCADE, the distribution of the 37 stations of the Grande array, and the small Piccolo cluster for fast trigger purposes are shown. The outer 12 clusters of the KASCADE array consist of $\mu$ - and $e / \gamma$-detectors, the inner 4 clusters of $e / \gamma$-detectors, only.

KASCADE-Grande stopped finally the active data acquisition of all its components end of 2012 and is presently being decommissioned. The collaboration, however, continues the detailed analysis of nearly 20 years of data. Moreover, with KCDC, the KASCADE Cosmic-ray Data Center, we plan to provide to the public the edited data via a customized web page.

The estimation of energy and mass of the primary particles is based on the combined investigation of the charged particle, the electron, and the muon components measured by the detector arrays of Grande and KASCADE. The multi-detector experiment KASCADE [1] (located at $49.1^{\circ} \mathrm{n}, 8.4^{\circ} \mathrm{e}$, 110 ma.s.l.) was extended to KASCADE-Grande in 2003 by installing a large array of 37 stations consisting of $10 \mathrm{~m}^{2}$ scintillation detectors each (fig. 1). KASCADE-Grande [2] provides an area of $0.5 \mathrm{~km}^{2}$ and operates jointly with the existing KASCADE detectors. While the Grande detectors are sensitive to charged particles, the KASCADE array detectors measure the electromagnetic component and the muonic component separately. These muon detectors enable to reconstruct the total number of muons on an event-by-event basis also for Grande triggered events.

\section{The all-particle energy spectrum}

In first steps of the data analysis, we reconstructed the all-particle energy spectrum. By combining both observables and using the hadronic interaction model QGSJet-II, a composition inde- 


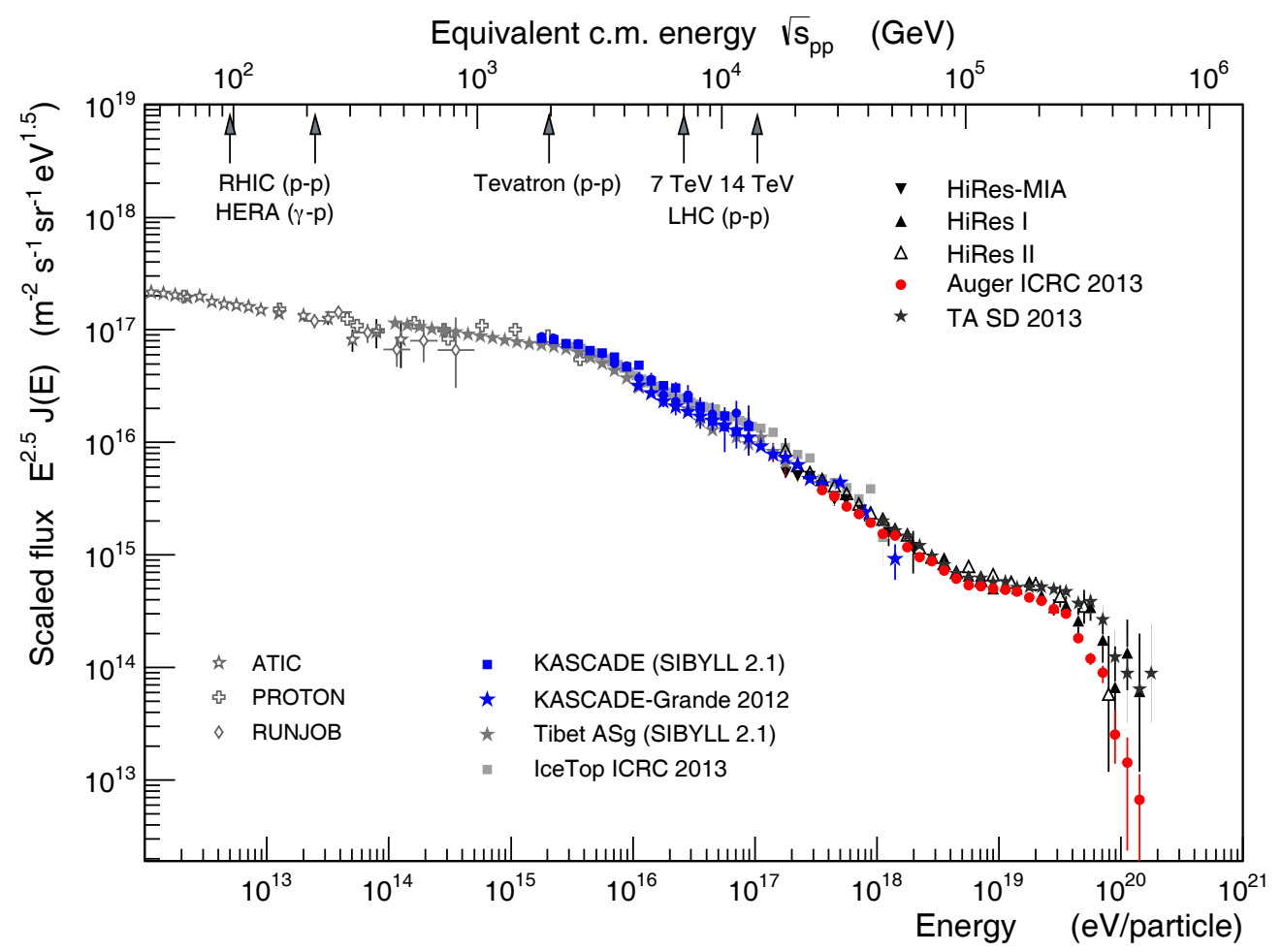

Figure 2: The all-particle energy spectrum obtained with KASCADE and KASCADE-Grande based on the QGSJet-II model and unfolded, i.e. corrected for the reconstruction uncertainties. Shown are the spectra in comparison with results of other experiments. In addition, the corresponding interaction energy at accelerators are indicated.

pendent all-particle energy spectrum of cosmic rays is reconstructed separately in the energy range of $10^{15} \mathrm{eV}$ to $10^{17} \mathrm{eV}$ for the KASCADE data and of $10^{16} \mathrm{eV}$ to $10^{18} \mathrm{eV}$ for the Grande data within a total uncertainty in flux of $10-15 \%[3,4,5]$.

Despite the overall smooth power law behavior of the resulting all-particle spectrum, there are some structures observed, which do not allow to describe the spectrum with a single slope index. Figure 2 shows the resulting all-particle energy spectra of various experiments. The justification of the 'knee' at a few times $10^{15} \mathrm{eV}$ is given since many years. But now, there is also a clear evidence that just above $10^{16} \mathrm{eV}$ the spectrum shows a 'concave' behavior, which is significant with respect to the systematic and statistical uncertainties. Another feature in the spectrum is a small break, i.e. knee-like feature at around $10^{17} \mathrm{eV}$. This slight slope change occurs at an energy where the rigidity dependent, i.e. charge dependent, knee of the iron component would be expected. At the highest energies, not in reach of the KASCADE-Grande experiment, two further features are established: the 'ankle' of the cosmic ray energy spectrum at a few times $10^{18} \mathrm{eV}$ and a strong suppression of the flux at around $5 \cdot 10^{19} \mathrm{eV}$.

\section{Composition}

The basic goal of the KASCADE-Grande experiment is the determination of the chemical composition in the primary energy range $10^{15}-10^{18} \mathrm{eV}$ by reconstructing individual mass group 
spectra. Structures observed in these individual spectra provide strong constraints to astrophysical models of origin and propagation of high-energy cosmic rays. For example, already in 2005 KASCADE could prove [3] (confirmed by different approaches [5]) that the knee is caused by a strong decrease of the light mass group of primary particles and not by heavy primary particles.

Meanwhile, with KASCADE-Grande we are able to investigate such individual mass group spectra also at higher primary energies. The main observables taken into account for these composition studies are the shower size $N_{c h}$ and the muon shower size $N_{\mu}$. Using the reconstruction of the energy spectrum by correlating $N_{c h}$ and $N_{\mu}$ on an event-by-event basis, the mass sensitivity is minimized by means of a parameter $k\left(N_{c h}, N_{\mu}\right)$. On the other hand, the evolution of $k$ as a function of energy keeps track of the evolution of the composition, and allows an event-by-event separation between light, medium and heavy primaries, at least. Using $k$ as separation parameter for different mass groups, where the values of $k$ have to be determined with help of simulations, directly the energy spectra of the mass groups are obtained [6, 7]. All the simulations for the described analyses are performed with the air-shower simulation package CORSIKA [8] allowing simulations based on various hadronic interaction models. The application of this methodical approach to shower selection and separation in various mass groups were performed and cross-checked in different ways, where figure 3, left panel, shows the main results:

Knee-like feature in the heavy component of primary cosmic rays: The reconstructed spectrum of the electron-poor events, i.e. the spectrum of heavy primaries, shows a distinct kneelike feature at about $8 \cdot 10^{16} \mathrm{eV}$. Applying a fit of two power laws to the spectrum interconnected by a smooth knee results in a statistical significance of $3.5 \sigma$ that the entire spectrum cannot be fitted with a single power law. The change of the spectral slope is $\Delta \gamma=-0.48$ from $\gamma=-2.76 \pm 0.02$ to $\gamma=-3.24 \pm 0.05$ with the break position at $\log _{10}(E / e V)=16.92 \pm 0.04$. Applying the same function to the all-particle spectrum results in a statistical significance of only $2.1 \sigma$ at the same energy and a change of the spectral slope from $\gamma=-2.95 \pm 0.05$ to $\gamma=-3.24 \pm 0.08$. Hence, the selection of heavy primaries enhances the knee-like feature that is already present in the all-particle spectrum.

Ankle-like feature in the light component of primary cosmic rays: An ankle-like feature is clearly visible in the spectrum of the electron-rich events, e.g. light elements of the primary cosmic rays at an energy of $10^{17.08 \pm 0.08} \mathrm{eV}$. At this energy, the spectral index changes by $\Delta \gamma=0.46$ from $\gamma_{1}=-3.25 \pm 0.05$ to $\gamma_{2}=-2.79 \pm 0.08$. Applying again a fit of two power laws to the spectrum results in a statistical significance of $5.8 \sigma$ that the entire spectrum cannot be fitted with a single power law. It is worth to mention that the changes in the spectrum of heavy primaries and in the spectrum of light elements are not connected by a bias in the separation or reconstruction procedures, which was checked in detail [10, 11, 9].

Influence of hadronic interaction models: It is crucial to verify the sensitivity of the observables to different primary particles and the reproducibility of the measurements with the hadronic interaction model in use as a function of sizes and the atmospheric depth. As it is well known from KASCADE data analysis [3] that the relative abundances of the individual elements or elemental groups are very dependent on the hadronic interaction model underlying the analyses, the strategy is to derive the energy spectra of the individual mass groups by applying different methods of composition analysis (see also [12,13]) and on basis of different hadronic interaction models $[14,11,9]$. Despite the fact, that the discussed spectrum is based on the QGSJet-II hadronic 

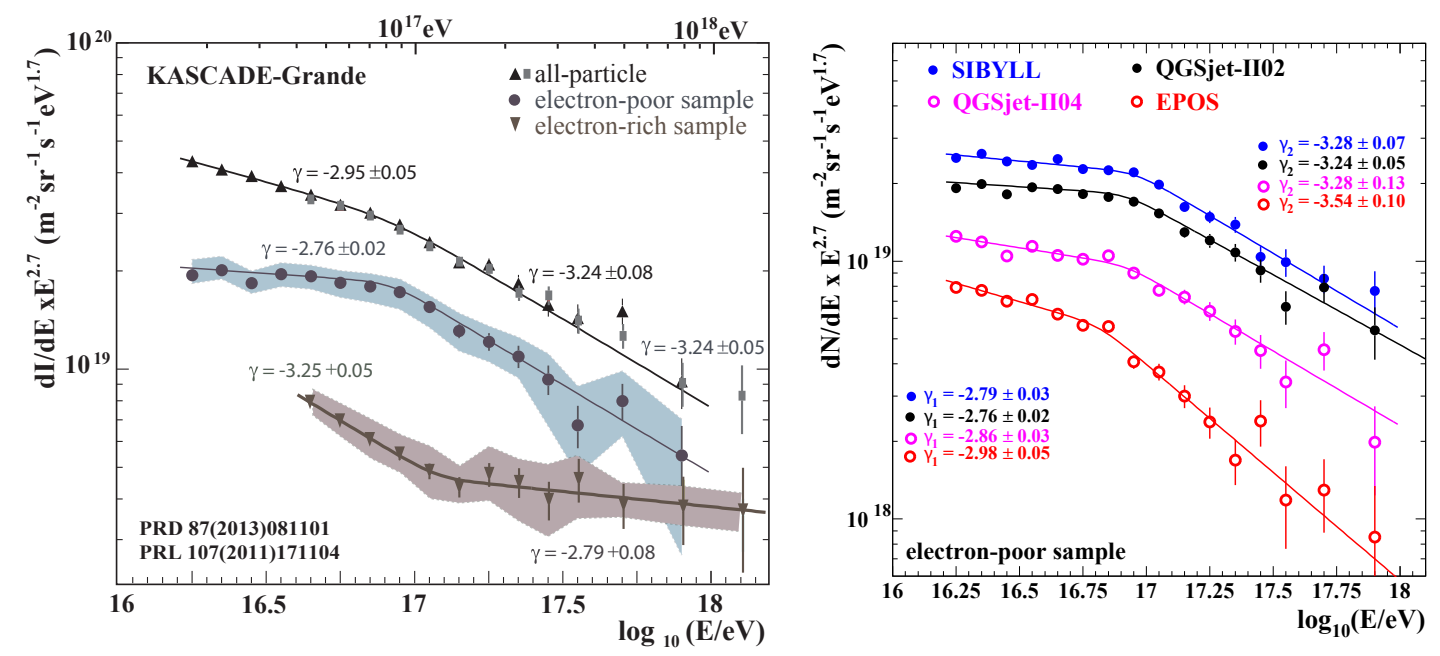

Figure 3: Left: All-particle, electron-poor, and electron rich energy spectra from KASCADE-Grande. The all-particle and heavy enriched spectrum is from ref. [6] and the all-particle and light primary spectrum results from a larger data set with higher energy threshold described in ref. [7]. Right: Reconstructed energy spectra of the heavy primary component for four hadronic interaction models. The error bars show the statistical uncertainties; fits on the spectra and resulting slopes before and after the heavy knee are also indicated [9].

interaction model [15], there is confidence that all the found structures of the energy spectrum remain stable [14]. Cross-checks of the general validity of the hadronic interaction models are performed within KASCADE-Grande by detailed investigations of the muon component (first results see $[16,17])$. The structure or characteristics of the spectra are found to be much less affected by the differences of the various hadronic interaction models than the relative abundances of the masses. An example is shown in figure 3, right panel, where the electron-poor spectra are displayed for the reconstruction on basis of four different hadronic interaction models.

\section{The KASCADE Cosmic-ray Data Center KCDC}

The KASCADE/KASCADE-Grande experiment was a large-area detector for the measurement of cosmic ray air showers lasting for more than 20 years and financed by taxes. The aim of this particular project is the installation and establishment of a public data center for high-energy astroparticle physics. In the research field of astroparticle physics, such a data release is a novelty, whereas the data publication in astronomy has been established for a long time. Therefore, there are no completed concepts, how the data can be treated/processed so that they are reasonably usable outside the collaboration. The first goal of KCDC is to make to the community the data from the KASCADE experiment available. A concept for this kind of data center (software and hardware) is already developed (fig. 4) and implemented, and will soon be opened as a platform to external users. The project faces thereby open questions, e.g. how to ensure a consistent calibration, how to deal with data filtering and how to provide the data in a portable format as well as how a sustainable storage solution can be implemented. In addition, access rights and license policy play a major role and have to be considered. KCDC is foreseen to be released in 2013. 

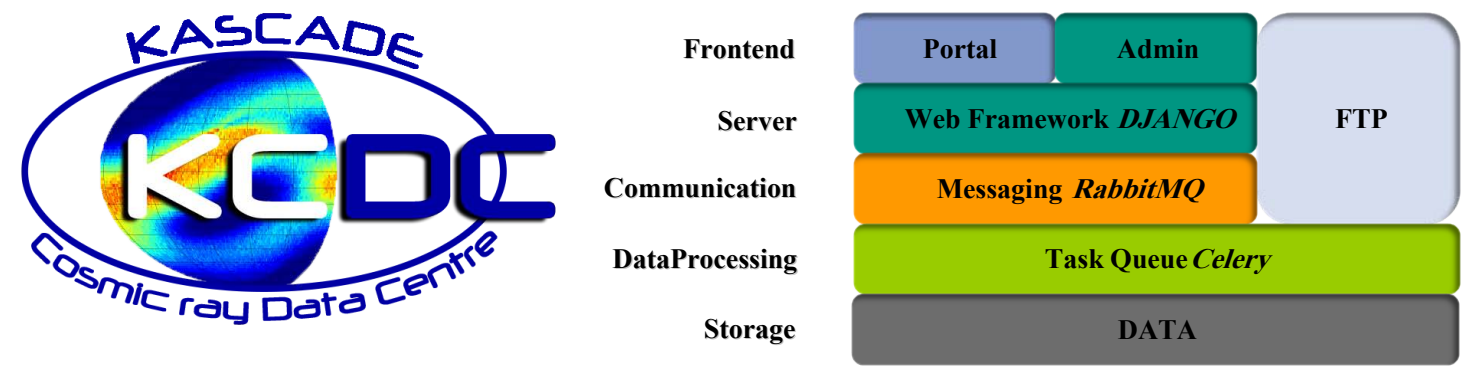

Figure 4: Logo and Software structure of KCDC, the KASCADE Cosmic-ray Data Center.

\section{Summary}

In summary, after separating the KASCADE-Grande measured events into a light and a heavy component, a knee-like feature is identified in the spectrum of the heavy component, and an anklelike feature is observed in the spectrum of the light component. Whereas the 'heavy-knee' occurs at an energy where the rigidity dependent knee of the iron component is expected, the 'lightankle' might indicate an early transition from galactic to extragalactic origin of cosmic rays. The KASCADE-Grande collaboration is ready to provide the entire data set to the public within the project KCDC, the KASCADE Cosmic Ray Data Center.

\section{References}

[1] T. Antoni et al. - KASCADE Coll., NIM A 513 (2003) 429.

[2] W.-D. Apel et al. - KASCADE-Grande Coll., NIM A 620 (2010) 202.

[3] T. Antoni et al. - KASCADE Coll., Astropart. Phys. 24 (2005) 1.

[4] W.-D. Apel et al. - KASCADE-Grande Coll., Astropart. Phys. 36, 183 (2012).

[5] W.-D. Apel et al. - KASCADE-Grande Coll., Astropart. Phys. 34, 476 (2011).

[6] W.-D. Apel et al. - KASCADE-Grande Coll., Phys. Rev. Lett. 107, 171104 (2011).

[7] W.-D. Apel et al. - KASCADE-Grande Coll., Phys. Rev. D 87, 081101(R)(2013).

[8] D. Heck et al., report Forschungszentrum Karlsruhe, FZKA 6019 (1998).

[9] M. Bertaina et al. (KASCADE-Grande Coll.), Proc. ICRC 2013, Rio de Janeiro, paper \#0196.

[10] S. Schoo et al. (KASCADE-Grande Coll.), Proc. ICRC 2013, Rio de Janeiro, paper \#0527.

[11] D. Kang et al. (KASCADE-Grande Coll.), Proc. ICRC 2013, Rio de Janeiro, paper \#0521.

[12] A. Chiavassa et al. (KASCADE-Grande Coll.), Proc. ICRC 2013, Rio de Janeiro, paper \#0092.

[13] D. Fuhrmann et al. (KASCADE-Grande Coll.), Proc. ICRC 2013, Rio de Janeiro, paper \#0531.

[14] W.-D. Apel et al. - KASCADE-Grande Coll., accepted at ASR 2013, doi:10.1016/j.asr.2013.05.008

[15] S. Ostapchenko, Phys. Rev. D 74, 014026/1-17 (2006).

[16] J.C. Arteaga-Velazquez et al. (KASCADE-Grande Coll.), Proc. ICRC 2013, Rio de Janeiro, paper \#0772.

[17] P. Łuczak et al. (KASCADE-Grande Coll.), Proc. ICRC 2013, Rio de Janeiro, paper \#0528. 